\title{
Optimized Tuned Deep Learning Model for Chronic Kidney Disease Classification
}

\section{R. H. Aswathy ${ }^{1, *}$, P. Suresh ${ }^{1}$, Mohamed Yacin Sikkandar ${ }^{2}$, S. Abdel-Khalek ${ }^{3}$, Hesham Alhumyani ${ }^{4}$, Rashid A. Saeed ${ }^{4}$ and Romany F. Mansour ${ }^{5}$}

\author{
${ }^{1}$ Department of Computer Science and Engineering, KPR Institute of Engineering and Technology, \\ Coimbatore, 641407, India \\ ${ }^{2}$ Department of Medical Equipment Technology, College of Applied Medical Sciences, Majmaah University, \\ Al Majmaah, 11952, Saudi Arabia \\ ${ }^{3}$ Department of Mathematics, College of Science, Taif University, Taif, 21944, Saudi Arabia \\ ${ }^{4}$ Department of Computer Engineering, College of Computers and Information Technology, Taif University, \\ Taif, 21944, Saudi Arabia \\ ${ }^{5}$ Department of Mathematics, Faculty of Science, New Valley University, El-Kharga, 72511, Egypt \\ *Corresponding Author: R. H. Aswathy. Email: rhaswathy@gmail.com \\ Received: 25 April 2021; Accepted: 06 June 2021
}

\begin{abstract}
In recent times, Internet of Things (IoT) and Cloud Computing (CC) paradigms are commonly employed in different healthcare applications. IoT gadgets generate huge volumes of patient data in healthcare domain, which can be examined on cloud over the available storage and computation resources in mobile gadgets. Chronic Kidney Disease (CKD) is one of the deadliest diseases that has high mortality rate across the globe. The current research work presents a novel IoT and cloud-based CKD diagnosis model called Flower Pollination Algorithm (FPA)-based Deep Neural Network (DNN) model abbreviated as FPA-DNN. The steps involved in the presented FPA-DNN model are data collection, preprocessing, Feature Selection (FS), and classification. Primarily, the IoT gadgets are utilized in the collection of a patient's health information. The proposed FPA-DNN model deploys Oppositional Crow Search (OCS) algorithm for FS, which selects the optimal subset of features from the preprocessed data. The application of FPA helps in tuning the DNN parameters for better classification performance. The simulation analysis of the proposed FPA-DNN model was performed against the benchmark CKD dataset. The results were examined under different aspects. The simulation outcomes established the superior performance of FPA-DNN technique by achieving the highest sensitivity of $98.80 \%$, specificity of $98.66 \%$, accuracy of $98.75 \%$, F-score of $99 \%$, and kappa of $97.33 \%$.
\end{abstract}

Keywords: Deep learning; chronic kidney disease; IoT; cloud computing; feature selection; classification

This work is licensed under a Creative Commons Attribution 4.0 International License, which permits unrestricted use, distribution, and reproduction in any medium, provided the original work is properly cited. 


\section{Introduction}

Internet of Things (IoT) is commonly employed in the integration of applications with computer systems. IoT is applied in a massive number of applications that consumes minimum energy, for example, refrigerator, smart watch, wrist band etc., rather than in high power consumption devices such as monitors, tablets, tools, mobile phones etc. In recent times, few home appliances like room freshener unit and air conditioners are operated by microcontroller along with sensing devices which result in practical outcomes. On a progressive note, when IoT is integrated with Cloud Computing (CC) model, it achieves high efficiency and is beneficial with massive number of standard features. In comparison with other sectors, clinical sector is one of the most promising areas that needs rapid development of clinical and sensing devices [1]. With medical devices getting costlier, most of the critical diseases are not predicted or treated at early stages. These are highly important and mandatory processes to save a human life. The proposed research is highly concentrated on Clinical Decision Support System (CDSS) through internet which applies IoT devices to verify the existence of severe diseases in human beings. IoT collects a number of details in terms of clinical applications whereas the data science paves an essential way to leverage the IoT for beneficial outcomes.

At present, Deep Learning (DL) models are implemented in various domains for pattern identification and new models are also being developed from the collected information. Machine Learning (ML) is one of the well-known and significant models in CDSS that can handle largescale data. Under the application of data analysis methods, data types can be defined in terms of Variety, Volume and Velocity. Neural Network (NN) is one of the effective classifiers [2] which invoke a novel technique with the help of CC and IoT methodologies to predict a patient's current state and disease condition. Healthcare details are collected and stored in significant UCI data repository whereas sensing gadgets are developed to broadcast the data on affected region for students in digital fashion. Different classification methods have been applied in the prediction of diseases in terms of F-measure, sensitivity and specification. This approach is effective in comparison with conventional models. Li et al. [3] deployed a novel energy method that can be operated in cloud-based IoT platform. It was developed to examine the images and videos recorded by a camera fixed on to the vehicles. This method is valuable in practical applications and computes the simulation by applying familiar simulation tool to observe the enhancements using IoT devices.

Stergiou et al. [4] evaluated the application of CC and IoT methods to solve the problems relevant to security. Tao et al. [5] deployed a new multi-layer cloud platform to enable proficient and prominent communication and multitasking compared to non-identical service generated by diverse agents in modern city. Moreover, ontology was created to solve the heterogeneity problems found in layer cloud platform. Kumar et al. [6] executed a new 3-tier structure for recording massive number of sensor details. Of the three layers, Tier-1 focuses on developing the data gathered from distinct sources. Then, Tier-2 deals with numerous sensor details saved in the cloud server. Finally, Tier-3 is a predictive model used for Heart Disease (HD) prediction. Dehury et al. [7] proposed a novel approach for real-time cloud management and technical data which is not related to IoT. Lee [8] introduced an efficient and useful Cyber Physical System (CPS) model for massive number of applications. Chen [9] labelled a modern framework in car camera system which applies mobile CC model in Deep Learning (DL) method. This framework is highly proficient in the identification of objects that exist in the recorded video while driving and is helpful in decision making process. Further, the data gets stored in cloud environment which 
reduces the usage of local storage space. Hence, this method is classified as Training, Recognizing, and Data collection model.

$\mathrm{Wu}$ [10] introduced a novel cloud-based model with ML technique for machinery prognostics. It makes use of Random Forest (RF) classifier to predict dry mill operations. Furthermore, RF model was presented under the application of MapReduce and was implemented on Amazon cloud environment. The proposed model ensured the application of RF classifier in accurate diagnosis of the disease. Muhammad et al. [11] conducted a research to track human voice pathology under the application of cloud and IoT. A new prediction approach was established on the basis of Local Binary Pattern (LBP) and was applied to observe human voice pathology. Gope et al. [12] presented a novel technique in body area sensor network on the basis of IoT devices. Using this model, the diseased person can be observed prominently with the help of compact and sensational sensor networks. Healthcare framework generally requires effective security mechanism. An online healthcare monitoring system was developed by Hossain et al. [13] to examine the health of patient and collect his or her details in order to find the reason behind death. Security methods such as watermarking and signal enhancements were combined in this study to remove the medical errors and find the existing attacks.

Significant developments that occurred recently in the medical domain of Chronic Kidney Diseases (CKD) have to be examined and such studies have to be conducted for a long time to understand disease prognosis. Some of the chronic illnesses associated with progressive CKD are stroke, diabetes, tumor, etc. Early prediction of chronic disease is important since it invokes a preventive measure for better treatment. Here, IoT and cloud-related CDSS are presented using computational science. With the application of IoT gadgets in medical field, the equipments are utilized frequently to gather details about healthcare parameters over a period of time and to verify the existence of abnormalities in short duration. Moreover, IoT and healthcare sensor measurements are employed effectively in the prediction of disease progression within a limited time interval. Personal medical services make use of IoT and cloud so that the lifetime can be expanded at minimum expense. Therefore, effective healthcare model is essential for disease examination using clinical devices.

The current research work presents a novel IoT and cloud-based CKD diagnosis model called Flower Pollination Algorithm (FPA)-based Deep Neural Network (DNN) model abbreviated as FPA-DNN. The presented FPA-DNN model comprises of data collection, preprocessing and Feature Selection (FS) as primary steps after which the classification is performed as the final step. Mainly, IoT gadgets are utilized to gather details about a patient's health. Afterward, the data is preprocessed following three ways namely, format conversion, data transformation and missing value replacement. In addition, the FPA-DNN model involves Oppositional Crow Search (OCS) algorithm for FS i.e., selection of optimal feature subset. The application of FPA helps in tuning the DNN parameters for better classification performance. The simulation analysis of the presented FPA-DNN model was conducted against CKD database and the experimental outcome is examined in detail in the upcoming sections.

\section{The Proposed FPA-DNN Technique}

The overall process of FPA-DNN is displayed in Fig. 1.

The presented FPA-DNN model comprises of data collection, preprocessing and Feature Selection (FS) as primary steps after which the classification is performed. The data collection component has the potential to collect essential details from CDS. Moreover, the collected data is 
pre-processed and FS steps enhance the supremacy of the information. Then, FPA-DNN method is implemented to train the OMDSS. In order to validate the approach, the projected FPA-DNN model is simulated through online mode with clinical data to check whether it can classify the data as either CKD or non-CKD.

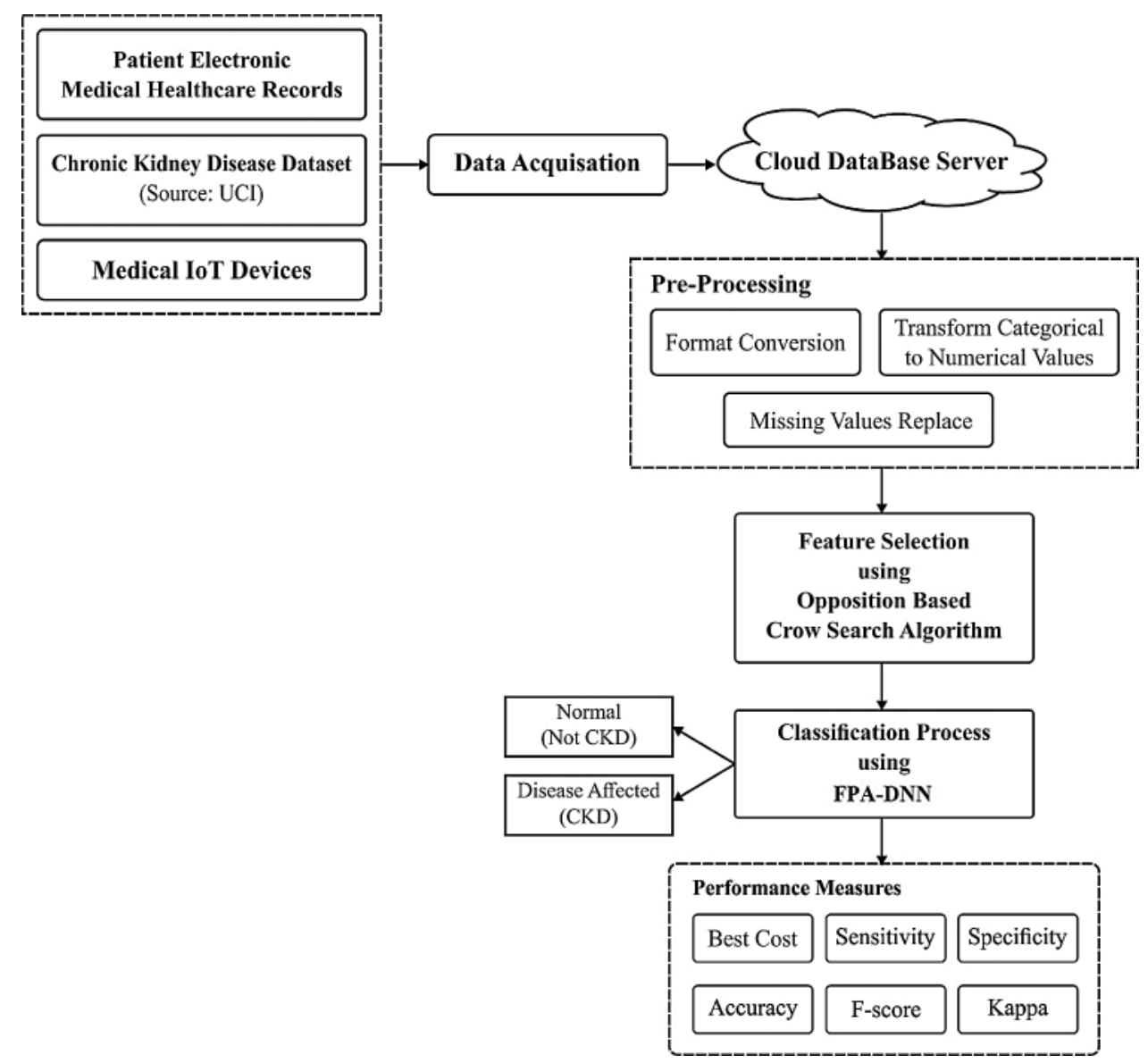

Figure 1: The overall process of the proposed FPA-DNN model

\subsection{Data Collection}

In this stage, three types of data are collected. The current framework enables the collection of patient information under the application of IoT devices. In general, the sensor linked to the human collects certain medical data for a limited duration. The newly-proposed OMDSS exploits $4 \mathrm{G}$ network for transferring the monitored data to Cloud Database Server (CDS).

\subsection{Preprocessing}

In this stage, the medical dataset is converted to a meaningful format under the application of three different ways. At first, the format conversion is performed during when the data is transformed from its actual format into .csv file format for further computations. Followed by, the categorical value in the database is converted to mathematical values such as ' 0 ' and ' 1 '. Finally, the missing values exist in the dataset are occupied by median model. 


\subsection{OCS Based FS Model}

In this stage, the optimal subset of features is selected using OCS algorithm from the preprocessed data. Raj et al. [14] introduced the CS model based on the natural hierarchy of crows in terms of hiding and food grabbing. It is also developed based on the structure of a flock, preservation of food hiding locations by crows, stealing the food, and following one another. By probability, a crow saves its caches from getting stolen. Both actual and novel locations of two crows are illustrated in Fig. 2. In order to make a CS model effective, a contrast operation is included in this model. For all initialized solutions, the adjacent opposite operation is initialized. When the solutions are compared, the best solution is selected as the optimal one. The execution strategy of OCS model is defined in a step-by-step manner herewith.

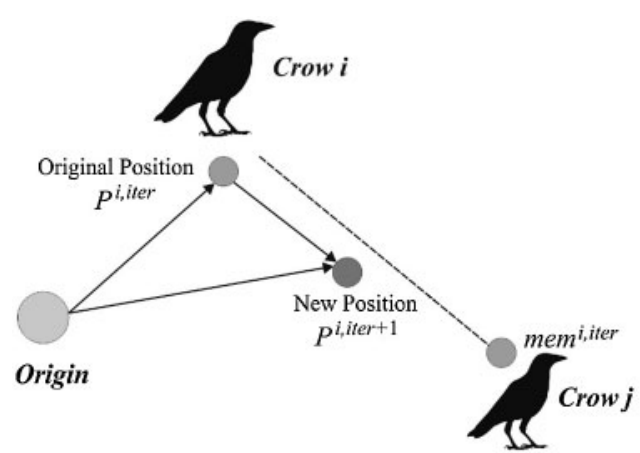

(a)

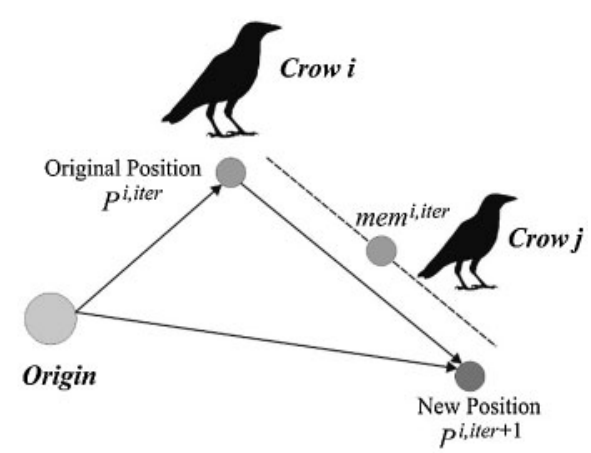

(b)

Figure 2: The general model of the CS representation

In population initialization stage, the population of crows is allocated by means of $F_{i}$, while the initialized crows (features) are randomly placed in a search area.

$F_{i}=F_{1}, F_{2}, \ldots, F_{n}, \quad$ where $i=1,2,3, \ldots, n$

In general, the meta-heuristic optimization methods are invoked with initial solutions which further attempts at enhancing a solution simultaneously. After comparing the solutions, the best one is decided as the initial solution. For sample, consider $f \in(g, h)$ which implies a real number. Using an opposite point definition, it is defined as follows.

$\tilde{f}_{j}=g_{j}+h_{j}-f_{j}$

Fitness Function (FF) of OCS approach is determined based on the objective function. In this model, the involvement is present to reach the best features from the applied database images.

$$
O F_{i}=M A X(\text { Accuracy })
$$

A crow forms a new position randomly by selecting a single crow among a flock of crows, where crow ' $\mathrm{j}$ ' should have its own position and memory space. The intervention position of a crow $P^{i, i t e r}$ is accomplished by the given Eq. (4).

$P^{i, i t e r+1}= \begin{cases}P^{i, \text { iter }}+r_{i} \times f^{i, \text { iter }} \times\left(\text { mem }^{j, \text { iter }}-P^{i, \text { iter }}\right) & \text { if } r_{j} \geq A P^{j, i t e r} \\ \text { randP } & \text { otherwise }\end{cases}$ 
The extension of Eq. (4) is defined as: $r_{i}$ and $r_{j}$ which are nothing but random values of crows, $i$ and $j$ correspondingly between [0 and 1], $f^{i, i t e r}$ implies the flight crow length $i, P$ signifies the place of a crow, mend refers to the memory position of $j^{\text {th }}$ crow and $A P^{j, \text { iter }}$ denotes the effective possibility of crow $j$ at iteration.

The recently updated crow's position and storage space values are improved using Eq. (5).

mem ${ }^{i, \text { iter }+1}= \begin{cases}P^{i, \text { iter }} & f\left(P^{j, \text { iter }+1}\right)>f\left(\text { mem }^{j, \text { iter }}\right) \\ \text { mem }^{i, i t e r} & \text { otherwise }\end{cases}$

It is observed that the fitness value for novel location of the crow is excellent in the applied location. The crow enhances the memory by new position. When maximum number of iterations is attained, the optimum position of the memory resembles the objective which is reported as the optimal solution of the extracted features. The flowchart of OCS model is shown in Fig. 3.

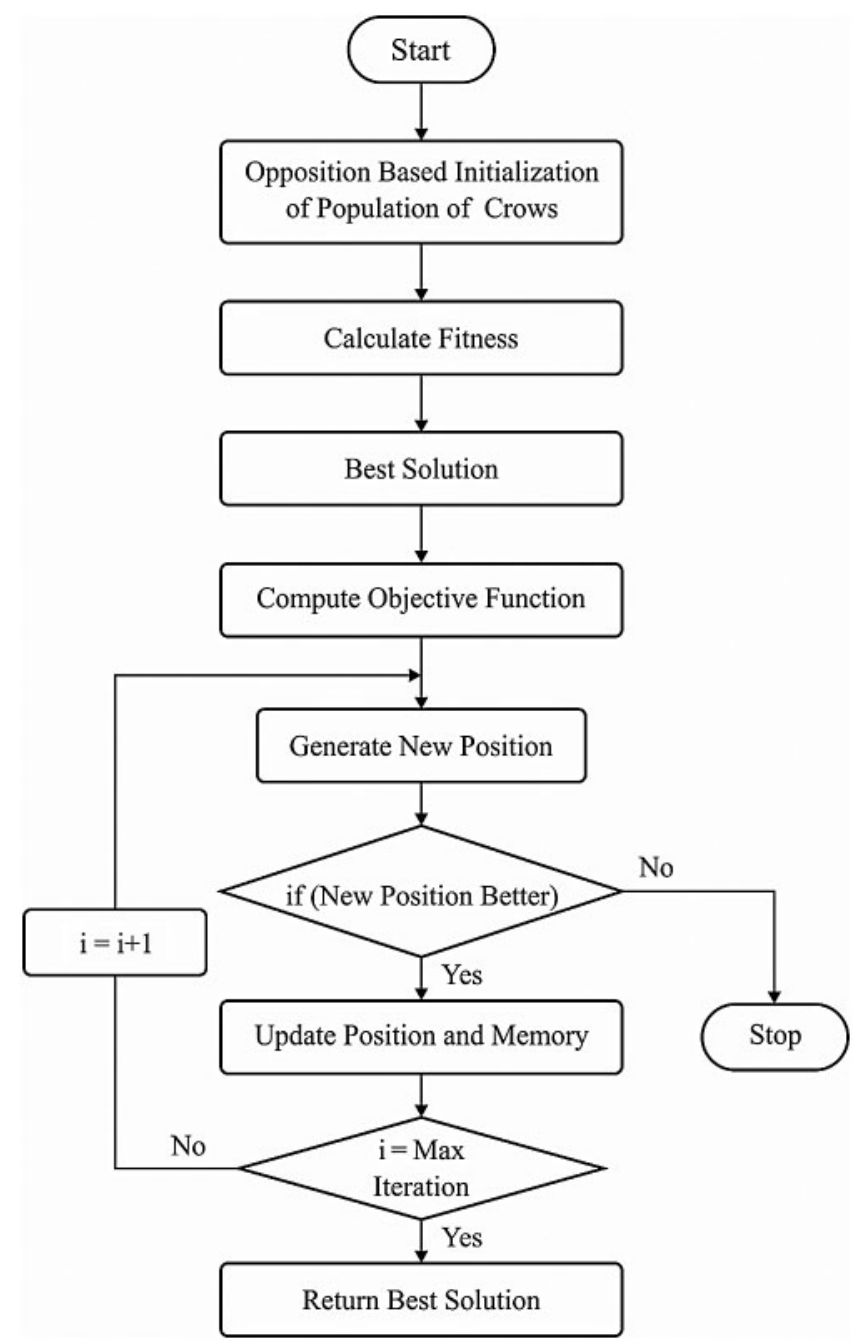

Figure 3: The flowchart of OCS 


\subsection{DNN Based Classification}

Generally, DL models are applied to achieve high dimension features from input database. Hence, the features gathered from DNN are used to improve the performance of classifiers. DNN classification model is applied prominently through the integration of a stack of auto encoders (AE) system, a result of application of SM classifier [15]. AE is comprised of input, hidden and output layers and is trained in an unsupervised manner to generate an equivalent input at consequent stage with limited erection error. Hence, the intensity of the outcome is similar to the intensity of the input. In addition, $A E$ is trained to embed the input into feature space which contributes a low dimension over input space. Thus, the dimensions of the code space are chosen as maximum over input space in order to improve the effectiveness of the classification in special events. Here, AE aims at providing the best presentation of the input vector by replacing proper codes.

Both input as well as output associations of an encoder are expressed as $c=g_{E}(W, b ; u)$ and is demonstrated as Eq. (6):

$\mathrm{c}=\mathrm{f}\left(\mathrm{b}+\mathrm{W}^{\mathrm{Z}} \mathrm{u}\right)$

where ' $\mathrm{f}$ ' denotes the activation function of an encoded neuron. Then, the weight of the encoding unit is represented as $\mathrm{W}$, which connects the input of hidden layer and b vector with neuron bias. The $\mathrm{u}$ vector is described as an input of encoder portion whereas vector $\mathrm{c}$ is meant to be the result of encoding unit. The correlation between input and output of the encoder is depicted herewith.

$\hat{\mathrm{u}}=\hat{\mathrm{f}}(\hat{\mathrm{b}}+\hat{\mathrm{Wc}})$

In this model, $\hat{\mathrm{f}}$ indicates the activation function of decoder neuron. The input output association of a decoder is expressed as $\hat{u}=g_{D}(\hat{W}, \hat{b} ; c)$. The result of $A E$ is illustrated by $\hat{\mathrm{u}}=\mathrm{g}_{\mathrm{AE}}(\mathrm{W}, \mathrm{b}, \hat{\mathrm{W}}, \hat{\mathrm{b}} ; \mathrm{u})$. The objective function of $\mathrm{AE}$ is defined as follows.

$\mathrm{E}_{\text {sparse }}=\mathrm{E}_{\mathrm{Z}}+\beta \sum_{\mathrm{q}=1}^{\mathrm{N}} \mathrm{KL}(\rho \| \hat{\rho})$

The pre-defined cost function is composed of two parts. At the initial stage, $\mathrm{E}_{Z}$ is meant to be an objective function of $\mathrm{NN}$, whereas $\beta$ denotes the weight of a sparsity penalty in Eq. (8):

$\mathrm{E}_{\mathrm{Z}}=\frac{1}{\mathrm{Z}} \sum_{\mathrm{k}=1}^{\mathrm{Z}} \mathrm{e}_{\mathrm{k}}^{2}+\frac{\lambda}{2}(\|\mathrm{~W}\|+\|\hat{\mathrm{W}}\|)$

where $\lambda$ denotes the regularization term which is highly used in the elimination of overfitting issues. Error vectors can be described as the difference between desired results and the actual output as showcased herewith.

$\mathrm{e}_{\mathrm{k}}=\left\|\mathrm{u}^{(\mathrm{k})}-\hat{\mathrm{u}}\right\|$ 
where $\mathrm{k}=1,2, \ldots, \mathrm{Z}$. It is an elegant model to observe that $\mathrm{E}_{\mathrm{Z}}$ denotes inner weight of the $\mathrm{AE}$ where,

$\mathrm{E}_{\mathrm{Z}}=\mathrm{E}_{\mathrm{AE}}(\mathrm{W}, \mathrm{b}, \hat{\mathrm{W}}, \hat{\mathrm{b}})$

The secondary part of Eq. (8) is expressed as follows.

$\mathrm{KL}\left(\rho \| \hat{\rho}_{\mathrm{q}}\right)=\rho \log \frac{\rho}{\hat{\rho}_{\mathrm{q}}}+(1-\rho) \log \frac{1-\rho}{1-\hat{\rho}_{\mathrm{q}}}$

where $\rho$ implies the sparsity value and $\hat{\rho}$ is provided below:

$\widehat{\rho_{\mathrm{j}}}=\frac{1}{\mathrm{Z}} \sum_{\mathrm{p}=1}^{\mathrm{Z}} \mathrm{f}_{\mathrm{q}}\left(\mathrm{u}^{(\mathrm{i})}\right)$

The AE components are linked together for developing a Stacked AE (SAE) system. The encoder is comprised of various AEs which are connected together for deploying SAE. By reforming input and output correlations of AE, the SAE network with L-cascaded AEs is accomplished as provided below.

$\mathrm{g}_{\mathrm{SAE}}=\mathrm{g}_{\mathrm{E}}^{1} \mathrm{og}_{\mathrm{E}}^{2} \mathrm{O} \cdots \mathrm{og}_{\mathrm{E}}^{\mathrm{L}}$

SAE approach is deployed by encoding unit of the AE, whereas the decoder parts of the AE are not used in the development of SAE. Finally, the training is completed by tuning the process which in turn improves the performance of DNN classifier under the application of FPA.

\subsection{FPA Based Tuning Process}

In FPA [16], pollination is a process carried out in plant generation principle. Flower and pollen gametes intend to offer a scalable solution to overcome specific optimization problems. Flower constancy is selected as the best solution and is a reliable one. In global pollination, the pollinator sends the pollen from long distance to higher fitting, whereas local pollination is performed in smaller regions with unique flower in shaded water. Global pollination is carried out with an opportunity termed as 'switch probability'.

If a stage is removed, the local pollination undergoes replacement. In FPA, four rules are applied in general which are given herewith.

- Live and cross-pollination are named as global ones in which the pollen pollinator makes use of the Levy flight method.

- Abiotic and self-pollination are meant to be local pollination.

- Insects are referred to as the Pollinators that are suitable in making flower fidelity.

- The interaction between global and local pollination is adjusted using a switch likelihood.

Therefore, both $1^{\text {st }}$ and $3^{\text {rd }}$ rules are described as follows.

$x_{q}^{\mathrm{t}+1}=x_{p}^{t}+\gamma \times L(\lambda) \times\left(g_{*}-x_{p}^{t}\right)$

where $x_{p}^{t}$ implies the pollen vector at iteration $t ; g_{*}$ denotes the recent optimal solution; $\gamma=\mathrm{a}$ refers to a scaling factor for managing step size; and $L$ represents the efficiency of pollination 
relevant to the step size for levy distribution [17]. Next, $L$ means a levy distribution as illustrated herewith.

$L \sim \frac{\lambda \times \Gamma(\lambda) \times \sin \frac{\pi \lambda}{2}}{\pi} \times \frac{1}{S^{1}+\lambda} S \gg S_{0} 0$

where $\Gamma(\lambda)$ represents a normal gamma function. Under local pollination, both $2^{\text {nd }}$ and $3^{\text {rd }}$ rules are applied as given herewith.

$x_{p}^{t+1}=x_{p}^{t}+\varepsilon\left(x_{q}^{t}-x_{k}^{\mathrm{t}}\right)$

where $x_{q}^{t}$ and $x_{k}^{t}$ denote the pollens from different flowers of the same plant.

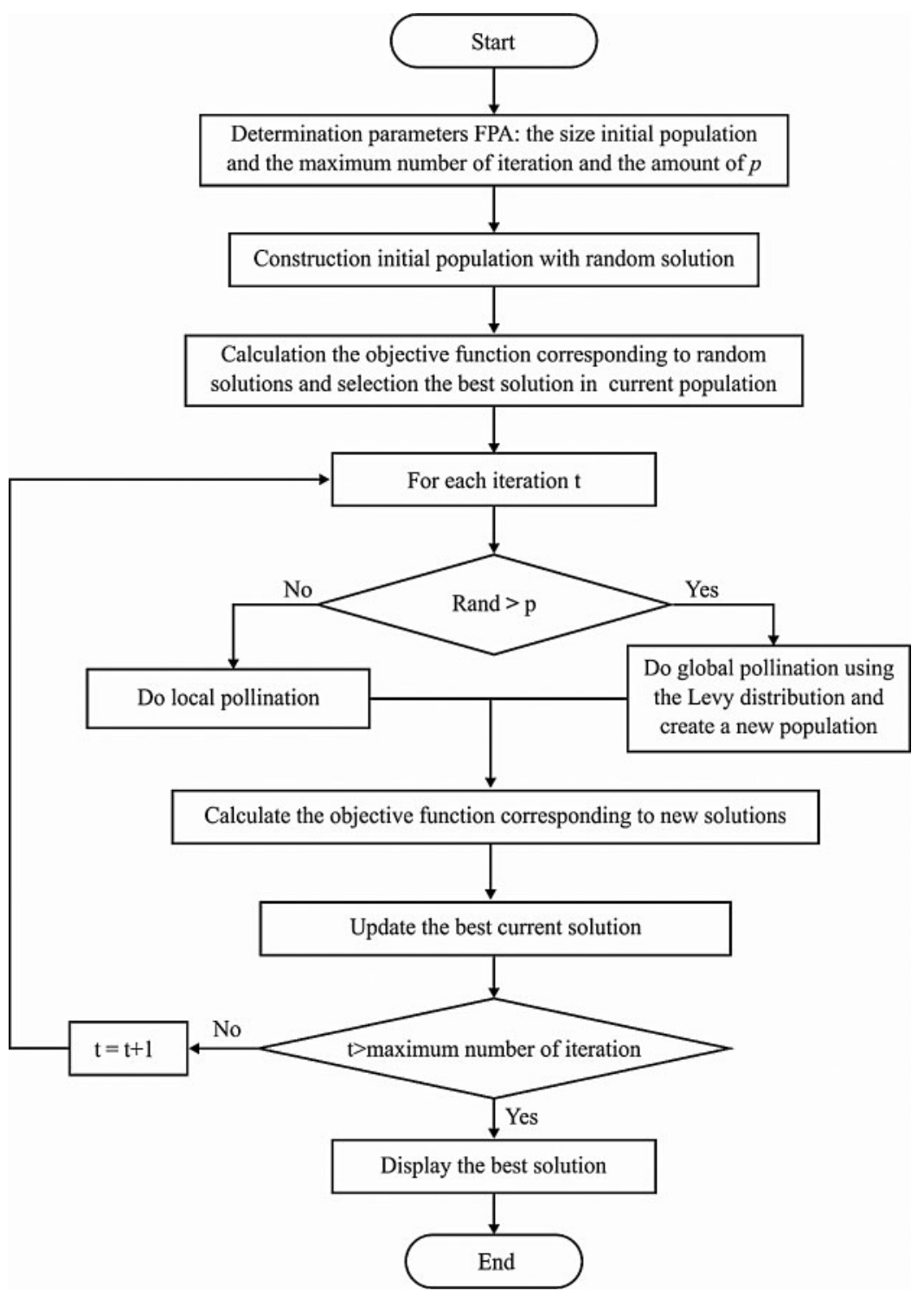

Figure 4: Flowchart of the FPA model 
In numerical form, if $x_{q}^{t}$ and $x_{k}^{\mathrm{t}}$ emerge from the same plant and are selected from similar population, then it is named as 'local random walk' whereas $\varepsilon$ is composed of uniform distribution from $[0,1]$. Fig. 4 demonstrates the workflow of FPA.

\section{Performance Validation}

The efficiency of the proposed FPA-DNN can be ensured by applying the model widely in different domains. The subsequent sections examine the dataset, estimation attributes and the accomplished outcomes. The simulation outcomes are applied with respect to diverse evaluation parameters.

\subsection{Dataset}

The outcomes of the FPA-DNN were investigated using a standard original CKD dataset. The dataset is composed of 400 instances with 24 features [18]. A collection of two classes i.e., positive and negative class labels are present in the database. For experimental investigation, 10fold cross validation method was applied.

\subsection{Results Analysis}

The results achieved by OCS-FS model were compared with existing FS models in terms of best cost. The chosen features are listed in Tab. 1 and Fig. 5. The table values portray that the CFS algorithm depicted an ineffective FS performance and attained the highest best cost of 0.79. Followed by, the PCA model demonstrated a moderate FS outcome with its best cost being 0.4570. Though the PSO-GS and GA-FS models achieved reasonable best costs such as 0.03656 and 0.03440 , the OCS-FS model outperformed other FS models with an optimal best cost of 0.00986. It is also displayed that the OCS-FS algorithm selected a set of 12 features out of 24, i.e., $2,4,5,8,10,12,13,14,16,19,20$, and 22 .

Table 1: The result analysis of FS methods for chronic kidney disease dataset

\begin{tabular}{lll}
\hline Methods & Best cost & Selected features \\
\hline OCS-FS & 0.00986 & $2,4,5,8,10,12,13,14,16,19,20,22$ \\
PSO-FS & 0.03656 & $15,12,24,23,13,20,11,8,18,3,9,1,14,5,2,6,17,19$ \\
GA-FS & 0.03440 & $16,24,13,9,14,17,22,19,2,15,23,18,12,6,4,10,3,20$ \\
PCA & 0.04570 & $1,2,3,4,5,6,7,8,9,10,11,12,13,14,15,16,17,18$ \\
CFS & 0.79000 & $4,6,7,10,15,17,19,22$
\end{tabular}

Fig. 6 shows the confusion matrix generated by FPA-DNN model during the execution time. From the figure, it is apparent that the FPA-DNN model classified a total of 248 and 147 instances under positive and negative classes respectively.

Fig. 7 shows the comparative analysis of FPA-DNN method against previous models [19-21] in terms of sensitivity and specificity. The figure implies that the Olex-GA approach performed poor in terms of diagnosis and reached a minimum sensitivity of $80 \%$ and specificity of $66.66 \%$. Along with this, the LR technology represented a better result i.e., sensitivity of $83 \%$ and specificity of $82 \%$. Moreover, the XGBoost approach showcased moderate and identical sensitivity and specificity values of $83 \%$ each. In line with this, the PSO technology produced considerable results with a sensitivity of $88 \%$ and a specificity of $80 \%$. Besides, the ACO approach attained 
an acceptable sensitivity of $88.88 \%$ and a specificity of $84.61 \%$. Moreover, the DT framework illustrated reasonable results with a sensitivity of $90.38 \%$ and a specificity of $89.28 \%$. Followed by, the MLP technology showcased a slightly moderate function with its sensitivity being $92.3 \%$ and specificity being $92.86 \%$. The FNC method, on the other hand, implied the least outcomes with a sensitivity of $95.68 \%$ and a specificity of $95.86 \%$. Furthermore, the D-ACO scheme depicted competent results with a sensitivity of $96 \%$ and a specificity of $93.33 \%$ respectively. Finally, the FPA-DNN framework provided supreme function with the highest sensitivity of $98.8 \%$ and specificity of $98.66 \%$.

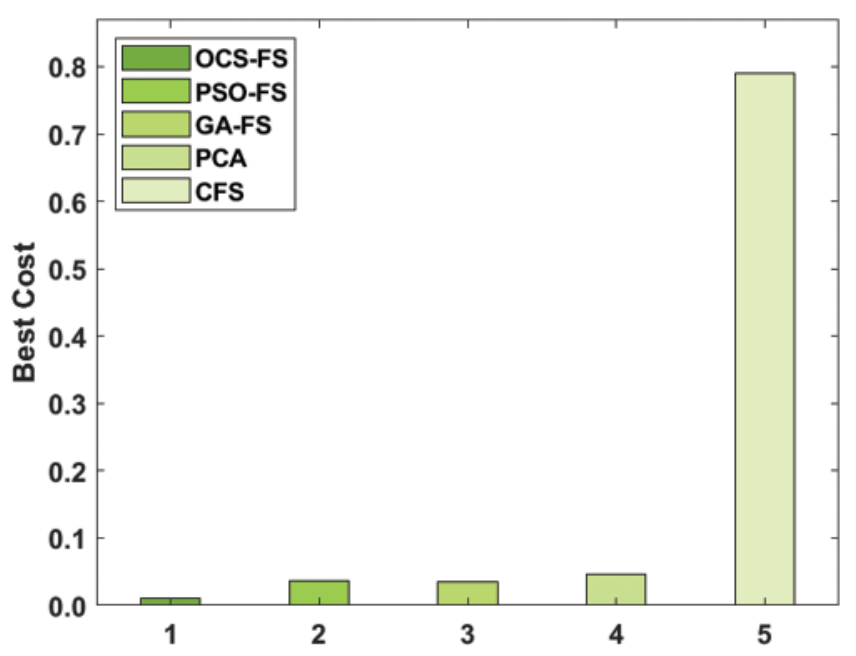

Figure 5: Best cost analysis of OCS-FS with other FS models

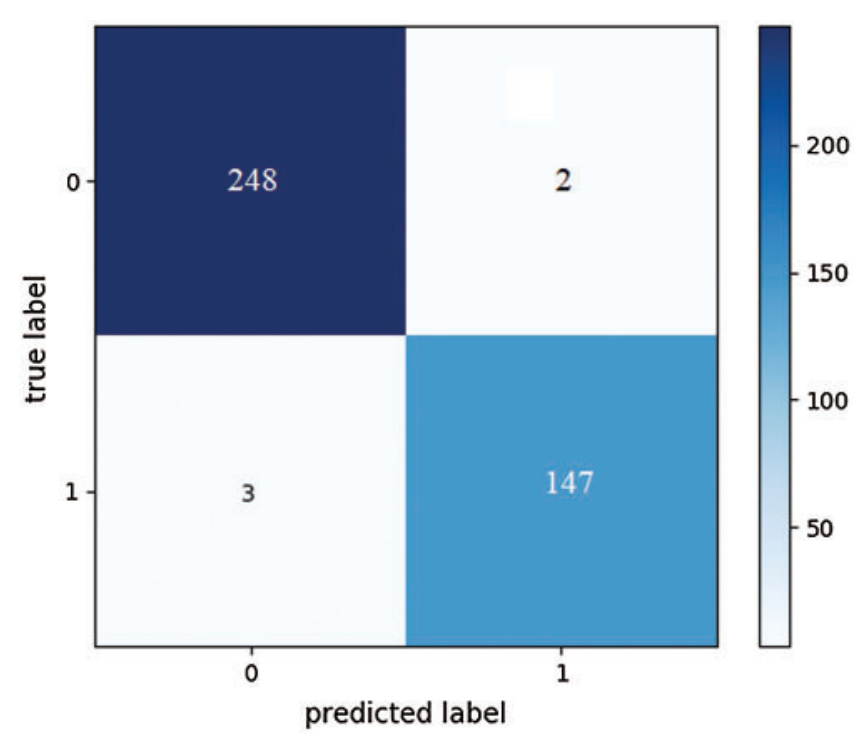

Figure 6: Confusion matrix of proposed FPA-DNN 


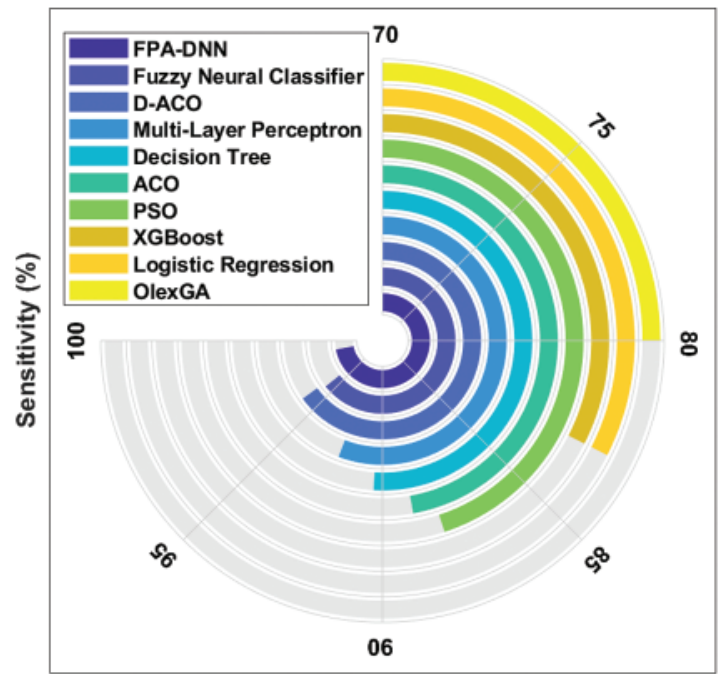

(a)

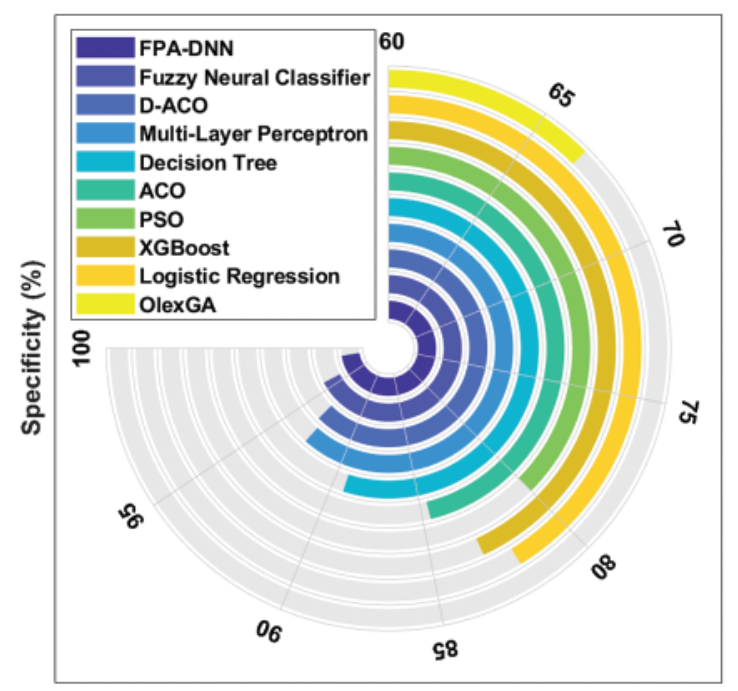

(b)

Figure 7: Results analysis of FPA-DNN with other models. (a) Sensitivity, (b) Specificity

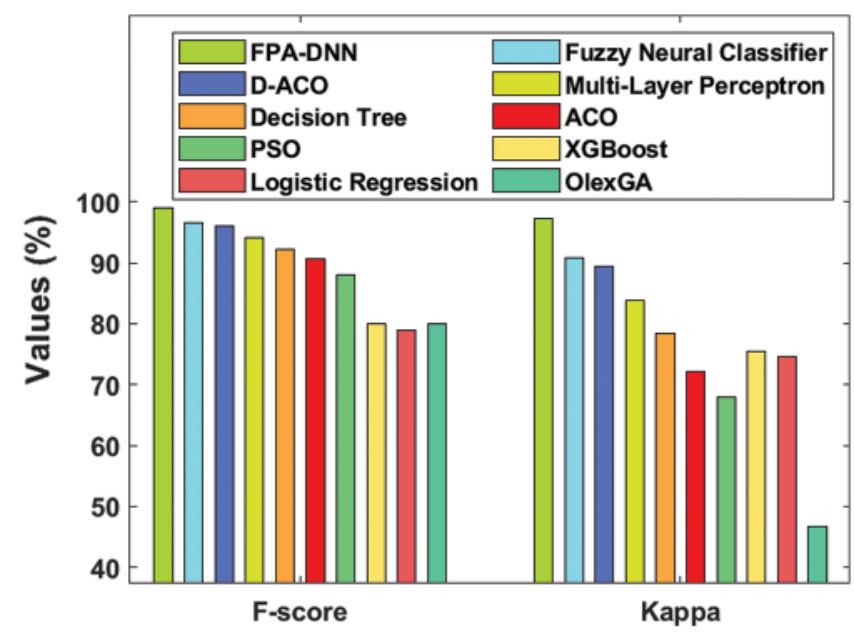

Figure 8: F-score and kappa analysis of FPA-DNN with existing models

Fig. 8 presents the results of comparative analysis of FPA-DNN technology against traditional models with respect to F-score and kappa. The figure portrays that the LR approach showcased ineffective diagnostics and accomplished a minimum F-score of $79 \%$ and a kappa of $74.6 \%$. Likewise, the OlexGA technique represented moderate results with an F-score of $80 \%$ and a kappa of $46.66 \%$. In addition, the XGBoost model depicted moderate F-score and kappa values such as $80 \%$ and $75.42 \%$ respectively. In line with this, the PSO model managed to represent a considerable outcome with an F-score of $88 \%$ and a kappa of $68 \%$. Then, the ACO scheme attained a better F-score of $90.56 \%$ and a kappa of $72.06 \%$. Moreover, the DT framework depicted acceptable results with an F-score of 92.15\% and a kappa of $78.37 \%$. Next, the MLP scheme showcased slightly improved results with an F-score of $94.11 \%$ and a kappa of $83.78 \%$. 
Additionally, the D-ACO method exhibited slightly better results with an F-score of $96 \%$ and a kappa of $89.33 \%$. The FNC model illustrated competing results with an F-score of $96.63 \%$ and a kappa of $90.87 \%$ respectively. Lastly, the FPA-DNN model outperformed all other methods by producing the maximum F-score of $99 \%$ and a kappa of $97.33 \%$.

Fig. 9 shows the results of accuracy analysis for the presented FPA-DNN model against other models. The figure showcases that the Olex-GA technique is a poor diagnostic performer as it attained the least accuracy of $75 \%$. Additionally, LR and XGBoost models displayed better accuracy values such as $82.0 \%$ and $83.0 \%$ correspondingly. Followed by, PSO and ACO techniques reached moderate accuracy values of $85 \%$ and $87.5 \%$ correspondingly. Besides, the DT and MLP models depicted reasonable performance with its accuracy values being 90\% and 92.5\% correspondingly. Moreover, D-ACO and FNC approaches demonstrated near optimal performance with accuracy values such as 95\% and 95.75\% correspondingly. At last, the FPA-DNN model offered a superior performance and achieved the highest accuracy of $98.75 \%$.

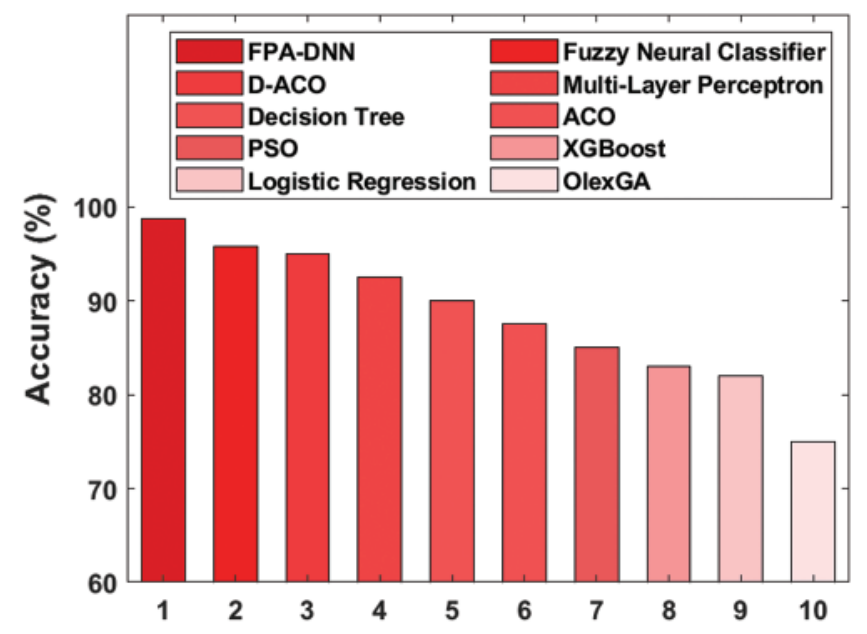

Figure 9: Accuracy analysis of FPA-DNN with existing models

\section{Conclusion}

The current research article designed a novel IoT and cloud-based CKD diagnosis model named FPA-DNN model. The presented FPA-DNN model comprises of different stages such as data collection, preprocessing, FS and classification. Primarily, IoT gadgets are utilized to gather details about a patient's health information. The FPA-DNN model makes use of OCS algorithm for FS in which an optimal subset of features is selected from the preprocessed data. The application of FPA helps in tuning the DNN parameters for better classification performance. The presented FPA-DNN model was experimentally evaluated against a benchmark CKD dataset. The obtained results verified that the FPA-DNN model is a superior performer as it achieved the highest sensitivity of $98.80 \%$, specificity of $98.66 \%$, accuracy of $98.75 \%$, F-score of $99 \%$, and kappa of $97.33 \%$. In future, the outcome of the FPA-DNN model can be enhanced using clustering and outlier removal techniques. 
Funding Statement: This research was supported by Taif University Researchers Supporting Project Number (TURSP-2020/216), Taif University, Taif, Saudi Arabia.

Conflicts of Interest: The authors declare that they have no conflicts of interest to report regarding the present study.

\section{References}

[1] L. Atzori, A. Iera and G. Morabito, "The internet of things: A survey," Computer Networks, vol. 54, no. 15 , pp. 2787-2805, 2010.

[2] P. Verma and S. K. Sood, "Cloud-centric IoT based disease diagnosis healthcare framework," Journal of Parallel and Distributed Computing, vol. 116, no. 15, pp. 27-38, 2018.

[3] Y. Li, A.-C. Orgerie, I. Rodero, B. L. Amersho, M. Parashar et al., "End-to-end energy models for edge cloud-based IoT platforms: Application to data stream analysis in IoT," Future Generation Computer Systems, vol. 87, no. 4, pp. 667-678, 2018.

[4] C. Stergiou, K. E. Psannis, B.-G. Kim and B. Gupta, "Secure integration of IoT and cloud computing," Future Generation Computer Systems, vol. 78, no. 54, pp. 964-975, 2018.

[5] M. Tao, J. Zuo, Z. Liu, A. Castiglione and F. Palmieri, "Multi-layer cloud architectural model and ontology-based security service framework for IoT-based smart homes," Future Generation Computer Systems, vol. 78, no. 6, pp. 1040-1051, 2018.

[6] P. M. Kumar and U. Devi Gandhi, "A novel three-tier internet of things architecture with machine learning algorithm for early detection of heart diseases," Computers \& Electrical Engineering, vol. 65, no. 4, pp. 222-235, 2018.

[7] C. K. Dehury and P. K. Sahoo, "Design and implementation of a novel service management framework for IoT devices in cloud," Journal of Systems and Software, vol. 119, no. 15, pp. 149-161, 2016.

[8] H. Lee, "Framework and development of fault detection classification using IoT device and cloud environment," Journal of Manufacturing Systems, vol. 43, no. 2, pp. 257-270, 2017.

[9] C. H. Chen, C. R. Lee and W. C. H. Lu, "Smart in-car camera system using mobile cloud computing framework for deep learning," Vehicular Communications, vol. 10, no. 6, pp. 84-90, 2017.

[10] D. Wu, C. Jennings, J. Terpenny and S. Kumara, "Cloud-based machine learning for predictive analytics: Tool wear prediction in milling," in 2016 IEEE Int. Conf. on Big Data (Big Data), Washington, DC, USA, pp. 2062-2069, 2016.

[11] G. Muhammad, S. M. M. Rahman, A. Alelaiwi and A. Alamri, "Smart health solution integrating IoT and cloud: A case study of voice pathology monitoring," IEEE Communications Magazine, vol. 55, no. 1, pp. 69-73, 2017.

[12] P. Gope and T. Hwang, "BSN-Care: A secure IoT-based modern healthcare system using body sensor network," IEEE Sensors Journal, vol. 16, no. 5, pp. 1368-1376, 2016.

[13] M. S. Hossain and G. Muhammad, "Cloud-assisted industrial internet of things (IIoT) -Enabled framework for health monitoring," Computer Networks, vol. 101, no. 6, pp. 192-202, 2016.

[14] R. J. S. Raj, S. J. Shobana, I. V. Pustokhina, D. A. Pustokhin, D. Gupta et al., "Optimal feature selection-based medical image classification using deep learning model in internet of medical things," IEEE Access, vol. 8, pp. 58006-58017, 2020.

[15] I. V. Pustokhina, D. A. Pustokhin, D. Gupta, A. Khanna, K. Shankar et al., "An effective training scheme for deep neural network in edge computing enabled internet of medical things (IoMT) systems," IEEE Access, vol. 8, pp. 107112-107123, 2020.

[16] X. S. Yang, "Flower pollination algorithm for global optimization," in UCNC 2012: Unconventional Computation and Natural Computation. Proc.: Lecture Notes in Computer Science (LNCS), New York City, NY, USA, vol. 7445, pp. 240-249, 2012.

[17] V. Porkodi, A. R. Singh, A. R. W. Sait, K. Shankar, E. Yang et al., "Resource provisioning for cyberphysical-social system in cloud-fog-edge computing using optimal flower pollination algorithm," IEEE Access, vol. 8, pp. 105311-105319, 2020. 
[18] UCI Machine Learning Repository, "Chronic Kidney Disease Data Set," 2020. [Online]. Available: https://archive.ics.uci.edu/ml/datasets/chronic_kidney_disease.

[19] P. Arulanthu and E. Perumal, "An intelligent IoT with cloud centric medical decision support system for chronic kidney disease prediction," International Journal of Imaging Systems and Technology, vol. 30, no. 3, pp. 815-827, 2020.

[20] M. Elhoseny, K. Shankar and J. Uthayakumar, "Intelligent diagnostic prediction and classification system for chronic kidney disease," Scientific Reports, vol. 9, no. 1, pp. 9583, 2019.

[21] K. Shankar, E. Perumal, M. Elhoseny and P. T. Nguyen, "An iot-cloud based intelligent computeraided diagnosis of diabetic retinopathy stage classification using deep learning approach," Computers, Materials \& Continua, vol. 66, no. 2, pp. 1665-1680, 2021. 